\title{
Desmopressin plus anticholinergic agent in the treatment of nocturnal enuresis: A meta-analysis
}

\author{
JIANYONG YU ${ }^{1 *}$, ZHAOJUN YAN $^{2 *}$, SHIYING ZHOU ${ }^{3}$, FENG HAN ${ }^{1}$, \\ FENG XIAO ${ }^{1}$, JIAN HAN ${ }^{4}$ and CONGLING SUN ${ }^{1}$ \\ ${ }^{1}$ Department of Pediatrics, Traditional Chinese Medicine Hospital of Yantai, Yantai, Shandong 264001; \\ ${ }^{2}$ Department of Pediatrics, Traditional Chinese Medicine Hospital of Shandong, Jinan, Shandong 250011; \\ ${ }^{3}$ Department of Pediatrics, Qilu Hospital of Shandong University, Qingdao, Shandong 266035; \\ ${ }^{4}$ Department of Pediatrics, Yantaishan Hospital of Yantai, Yantai, Shandong 264001, P.R. China
}

Received July 19, 2016; Accepted May 5, 2017

DOI: 10.3892/etm.2017.4897

\begin{abstract}
The present study aimed to evaluate the clinical efficacy and safety of combination therapy comprising desmopressin plus anticholinergic agent compared with desmopressin alone for children with nocturnal enuresis (NE). A meta-analysis of 8 eligible studies was performed to analyze the effects of desmopressin plus anticholinergic agent combination therapy and desmopressin monotherapy in the treatment of NE in children. The overall odds ratio (OR) or standardized mean difference (SMD) and 95\% confidence interval were calculated for full responders (FR), partial responders (PR), non-responders (NR), the change in the mean number of wet nights and adverse events. Following 1 month of treatment, efficacy analysis yielded an OR of 3.736, which suggested that the proportion of FR for patients treated with the combination therapy was higher than that for patients treated with monotherapy. Analysis of the change in the mean number of wet nights yielded an SMD of 0.719, which indicated that the change in the mean number of wet nights in the patients treated with combination therapy was greater than that in the patients treated with monotherapy. Following 3 months of treatment, the OR calculated for FR plus PR compared with NR was 2.857, indicating that the proportion of FR and PR was elevated by the combination therapy compared with desmopressin alone. The OR for adverse events was 4.074 , which suggested that the combination therapy did not lead to more adverse events in the treatment of NE. Therefore, the present meta-analysis suggests that, compared with desmopressin monotherapy, a combination
\end{abstract}

Correspondence to: Ms. Congling Sun, Department of Pediatrics, Traditional Chinese Medicine Hospital of Yantai, 39 Xingfu Road, Yantai, Shandong 264001, P.R. China

E-mail: suncongling_sd@sina.com

*Contributed equally

Key words: desmopressin, anticholinergic agent, combination therapy, nocturnal enuresis in children, meta-analysis therapy comprising desmopressin and anticholinergic agent is more effective with equivalent safety for children with NE.

\section{Introduction}

Nocturnal enuresis (NE), defined as intermittent incontinence of urine during sleep, is ubiquitous in newborns $(1,2)$. Previous studies have demonstrated that $15-20 \%$ of 5-year-old children, $5 \%$ of 10 -year-olds, $1-2 \%$ of individuals aged 15 years and $2 \%$ of young adults also suffer from NE, which is a persistent and prevalent problem posing considerable inconvenience for children and their parents $(3,4)$. According to the World Health Organization classification, from the age of 5 years, NE is regarded as a pathological disorder (5). This pathological disorder occurs in the absence of congenital or acquired defects of the nervous system (6) and it appears that a mismatch between bladder capacity and nocturnal urine production is the primary pathogenesis of NE (7).

Desmopressin, one of the first line treatments for NE, is an analog of the naturally produced vasopressin, and has the effect of reducing nocturnal urine production (7). This evidence-based treatment is most efficient in children with nocturnal polyuria and normal bladder reservoir function, but retains a success rate of $\sim 50 \%$ in patients who exhibit nonmonosymptomatic NE with increased voiding frequency, daytime incontinence or urgency $(8,9)$. It was reported that patients with monosymptomatic NE (MNE) who received desmopressin treatment attained $85 \%$ dry nights after 3 months (10). A clinical trial also suggested that with desmopressin therapy, the full and partial response rates were 26.3 and $43.5 \%$, respectively, for children with primary MNE (PMNE) (11). However, the clinical outcome of desmopressin monotherapy is not always satisfactory specifically for those patients with severe bedwetting refractory and a small functional bladder capacity (2).

It has been documented that anticholinergic agents, or parasympathetic nervous system antagonist drugs, serve an important role in increasing functional bladder capacity (6). The mismatch between nocturnal urine production and bladder storage capacity may result from a reduction in nocturnal functional bladder capacity (2). Therefore, 
anticholinergic agents, such as tolterodine, oxybutynin or propiverine may be introduced for the treatment of NE. A randomized double-blind placebo-controlled trial performed by Austin et al (12) indicated that for children who exhibit no response to desmopressin for MNE, a marked reduction in the mean number of wet nights was observed when treatment with desmopressin plus an anticholinergic was administered, compared with treatment with desmopressin alone. Therefore, the present meta-analysis was conducted to comprehensively estimate the efficacy and safety of the combination therapy of desmopressin plus an anticholinergic agent compared with the first line regimen desmopressin alone in the treatment of patients with NE.

\section{Materials and methods}

Search strategy. The current study performed three-rounds of selection to identify the studies for this meta analysis. For the first-round search, multiple databases including PubMed (https://www.ncbi.nlm.nih.gov/pubmed), Global Cross-database (https://www.ncbi.nlm.nih.gov/sites/gquery), MEDLINE (https://www.nlm.nih.gov/) and Google Scholar (https://scholar.google.com) were searched for studies evaluating the clinical efficacy and safety of desmopressin in the treatment of NE. Key search terms included 'nocturnal enuresis' and 'desmopressin' and 'anticholinergic'. Only studies published prior to July 15, 2015 were included. The inclusion criteria were as follows: i) Patients in the case group were treated with a combination therapy of desmopressin and an anticholinergic agent while participants in the control group were treated with desmopressin monotherapy alone or plus placebo, ii) the age of patients ranged from 5 to 18 years, since NE is not diagnosed in children $<5$ years and persons $>18$ years of age are not classed as children $(13)$, iii) the treatment duration was $\geq 2$ weeks, and iv) symptom information such as frequency of wet nights or maximum voided volume was documented. The exclusion criteria were as follows: i) Children with known contraindications to NE treatment, as assessed by a doctor, ii) patients aged $<5$ years and $>18$ years, and iii) a combination therapy of desmopressin plus other drugs (not anticholinergic agents).

Data extraction. The potentially eligible studies according to the inclusion and exclusion criteria were assessed by two authors, independently. The reference lists were also examined manually to identify potentially relevant literature. Unpublished reports were not considered. If some studies were overlapping, only those containing the most extensive results were included. The following information was extracted from the covered studies: The first author, population and type of patients, the age and sex ratio of patients, the number of participants in each group and the detailed regimen for each group. Furthermore, data regarding wet nights and adverse events, including nasal irritation, headache and mouth dryness, were also collected for the present detailed analysis.

Statistical analysis. The present study used STATA software, version 12 (StataCorp LLC, College Station, TX, USA) for statistical analysis and $\mathrm{P}<0.05$ was considered to represent a statically significant difference. In the current study, the efficacy of the therapy was evaluated using International Children's Continence Society (ICCS) criteria at 1 and 3 months after the start of treatment (14), which are also cited by other relevant studies $(2,15-17)$. According to the ICCS, patients were defined as full responders (FR; 90-100\% reduction in wet nights following treatment compared with baseline), partial responders (PR; 50-90\% reduction) and non-responders (NR; $<50 \%$ reduction) (14). Patients were categorized with regard to clinical outcomes as FR+PR vs. NR, and FR vs. PR+NR. The change in the mean number of wet nights $(18,19)$ was also measured to assess the efficacy of the therapy, and the current study defined the change in the mean number of wet nights as the difference in the number of wet nights observed prior to and following treatment. The safety of the therapy was estimated using adverse events, which included nasal irritation, headache and mouth dryness.

In the present meta-analysis, for dichotomous data [clinical outcomes: FR+PR vs. NR, FR vs. PR+NR, and adverse events], the overall odds ratio (OR) and $95 \%$ confidence interval $(\mathrm{CI})$ were calculated while the standardized mean difference (SMD) and 95\% CI were estimated for continuous data (mean number of wet nights). A previously described $\mathrm{I}^{2}$ index (20) was applied for evaluation of the heterogeneity among studies. The Mantel-Haenszel (M-H) fixed-effects model (21) was applied to calculate the $\mathrm{I}^{2}$ index. When the $\mathrm{I}^{2}$ index was $<50 \%$ or $\mathrm{P}>0.1$, the $\mathrm{M}-\mathrm{H}$ fixed-effects model was adopted for dichotomous data to calculate the overall OR and 95\% CI, whereas the Inverse-Alteration (I-V) fixed-effects model (22) was used for continuous data to calculate the SMD and 95\% CI. Otherwise, the DerSimonian and Laird (D-L) random-effects model (23) was selected for dichotomous and continuous data for the calculation of the overall OR or SMD and 95\% CI.

For the calculation of the overall OR or SMD and 95\% CI, the data in the desmopressin monotherapy group (control group) served as a control. For the FR+PR vs. NR comparison, an OR $>1$ signified that the proportion of FR and PR in the combination therapy group was higher than that in the desmopressin monotherapy group and the combination therapy was more effective for the treatment of NE. For the FR vs. PR+NR comparison, an OR $>1$ suggested that the proportion of FR in the combination therapy group was higher than that in the desmopressin monotherapy group and the combination therapy was more effective. For the change in the mean number of wet nights, a SMD $>0$ indicated that the change was more evident in the combination therapy group than the desmopressin monotherapy group and the combination therapy had a more effective clinical outcome. For adverse events, an OR $>1$ signified that more adverse events occurred in the combination therapy group. Forest plots were generated to summarize the results. Begg's funnel plots and Egger's test (24) were used to examine publication bias, where noticeable asymmetry of the funnel plots indicates the existence of marked publication bias.

In Table II, we defined 'Change 1' or 'Change 2' into 'Change: Mean \pm standard deviation (SD)'. Therefore, the formula for calculation of 'Change: Mean \pm SD' of Table II is: (mean1-mean2) $\pm \sqrt{ }\left(\mathrm{SD}^{2}+\mathrm{SD} 2^{2}-2 \times \mathrm{R} \times \mathrm{SD} 1 \times \mathrm{SD} 2\right)$. In detail, $\mathrm{R}$ is 0.5 , and mean1 and SD1 are the values obtained prior to treatment and mean 2 and SD2 are the values following treatment. For example, 'Change $1(6.60 \pm 6.86)$ ' $=$ [The values of prior to treatment (13.30). The values of First treatment period 


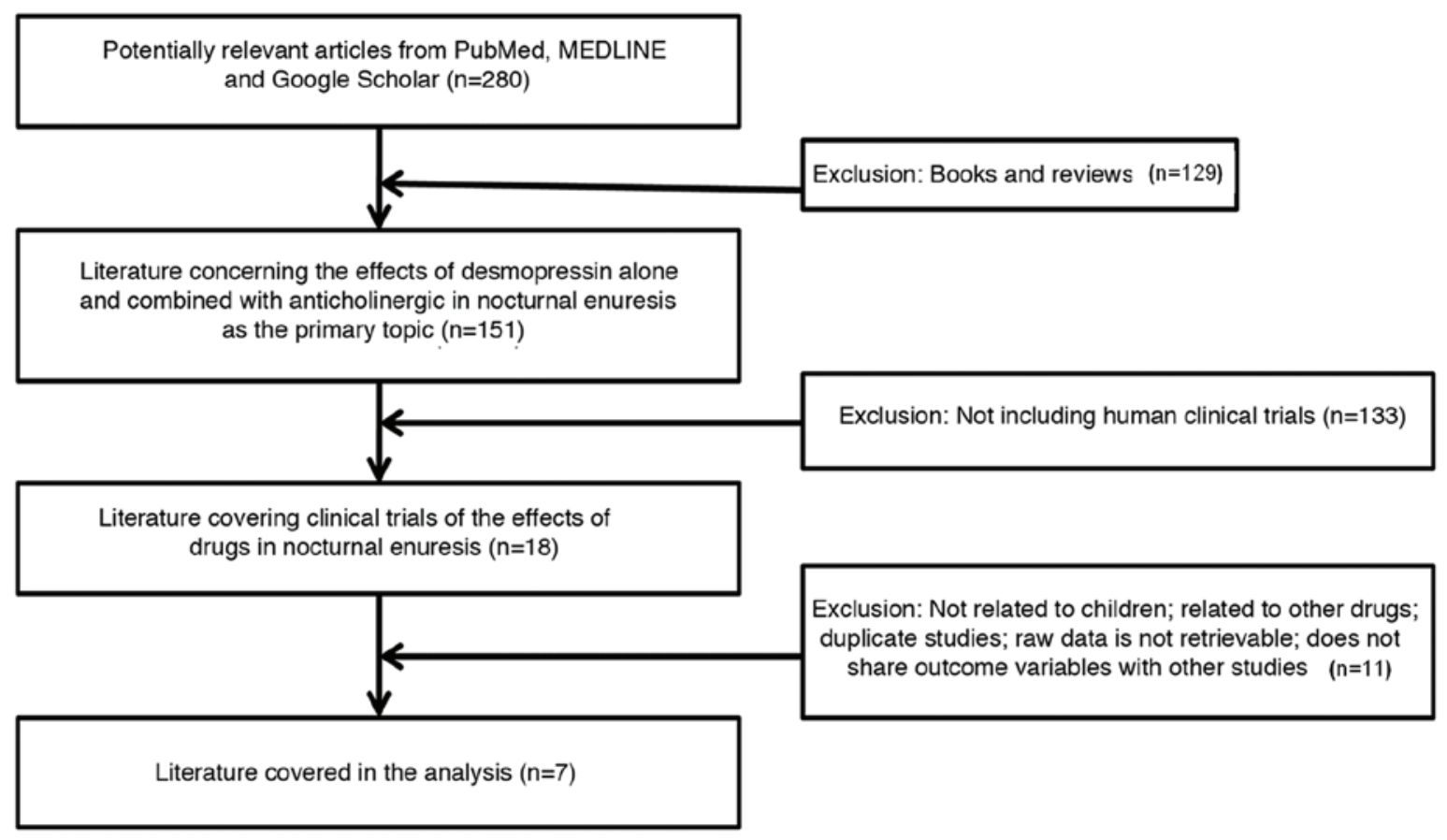

Figure 1. Flow chart of study selection and specific reasons for exclusion from the meta-analysis.

$(6.70)] \pm \sqrt{ }\left(\mathrm{SD}^{2}\left(3.40^{2}\right)+\mathrm{SD} 2^{2}\left(7.90^{2}\right)-2 \times \mathrm{R}(0.5) \times \mathrm{SD} 1\right.$ (3.40) x SD2 (7.90).

\section{Results}

Study characteristics. A total of 280 papers were retrieved following the first search, and 273 of them were eliminated from the analysis for reasons shown in Fig. 1. A total of 7 articles, covering 8 studies $(2,3,12,15,17,19,25)$ met the inclusion criteria; Triantafyllidis et al (3) contains two separate studies with $5 \mathrm{mg}$ oxybutinine or $2 \mathrm{mg}$ tolteodine. The primary characteristics of the 8 eligible studies are presented in Table I. The mean number of wet nights prior to and following treatment (at 1 and 3 months) is presented in Table II.

Evaluation of $F R+P R$ vs. NR following 1 and 3 months of treatment. For the analysis of FR+PR vs. NR following treatment, 6 eligible studies were included for 1 month of treatment and 5 eligible studies were included for 3 months of treatment. The results are presented in Table III. The M-H fixed-effects model was applied for the calculation of overall OR and $95 \% \mathrm{CI}$, as there was no significant heterogeneity (1 month, $\mathrm{I}^{2}=15.90 \% ; 3$ months, $\left.\mathrm{I}^{2}=45.60 \%\right)$. Following 1 month of treatment, the overall OR was 1.350 (95\% CI: 0.93-1.98, $\mathrm{P}=0.12$; Table III and Fig. 2A). No significant difference was observed for FR+PR vs. NR between desmopressin plus anticholinergic agent combination therapy and desmopressin alone in the treatment of patients with NE, and the ratio of FR+PR to NR in the combination therapy group was similar to that in the monotherapy group following 1 month of treatment. However, following 3 months of treatment, the overall OR was 2.86 (95\% CI: 1.51-5.41, P=0.001; Table III and Fig. 2B). A significant difference was identified for FR+PR vs. NR between the two therapies $(\mathrm{P}<0.05)$ and the ratio of $\mathrm{FR}+\mathrm{PR}$ to $\mathrm{NR}$ in the combination therapy group was significantly higher than that in the monotherapy group at 3 months after treatment $(\mathrm{P}<0.05$; Table III). The statistical analysis of FR+PR vs. NR demonstrated that, compared with monotherapy the ratio of FR+PR to NR in the combination therapy group was significantly increased when the treatment duration was 3 months and greater higher numbers of FR+PR were observed for long-term treatment with the combination therapy than for short-term treatment.

Evaluation of FR vs. $P R+N R$ following 1 and 3 months of treatment. For the analysis of FR vs. PR+NR following treatment, 5 eligible studies were included at 1 month and 3 eligible studies were included at 3 months. The primary results are presented in Table III. Considering the value of the $\mathrm{I}^{2}$ index ( 1 month, $\mathrm{I}^{2}=0.00 \%$; 3 months, $\mathrm{I}^{2}=68.20 \%$ ), the $\mathrm{M}-\mathrm{H}$ fixed-effects model was used to calculate the overall OR and $95 \% \mathrm{CI}$ for 1 month, while the D-L random-effects model was used for 3 months. Following 1 month of treatment, the overall OR was 3.74 (95\% CI: 2.11-6.63, P<0.001; Table III and Fig. 3A), demonstrating that there was significant difference in FR vs. PR+NR between the combination therapy and monotherapy, and the ratio of FR to $\mathrm{PR}+\mathrm{NR}$ in the combination therapy group was significantly higher than that in the monotherapy group at 1 month after treatment. However, following 3 months of treatment, the overall OR was 2.73 (95\% CI: 0.48-15.55, $\mathrm{P}=0.26$; Table III and Fig. 3B), implying that there was no significant difference in FR vs. PR+NR between the two therapies and the ratio of FR to $\mathrm{PR}+\mathrm{NR}$ in the patients treated with combination therapy was similar with that in the patients treated with monotherapy at 3 months after treatment. The statistical analysis of FR vs. PR+NR suggested that, when compared with monotherapy, the ratio of FR achieved with combination therapy was elevated significantly when the treatment duration was 1 month, and more FR were observed for short-term treatment with the combination therapy. Considering the results 


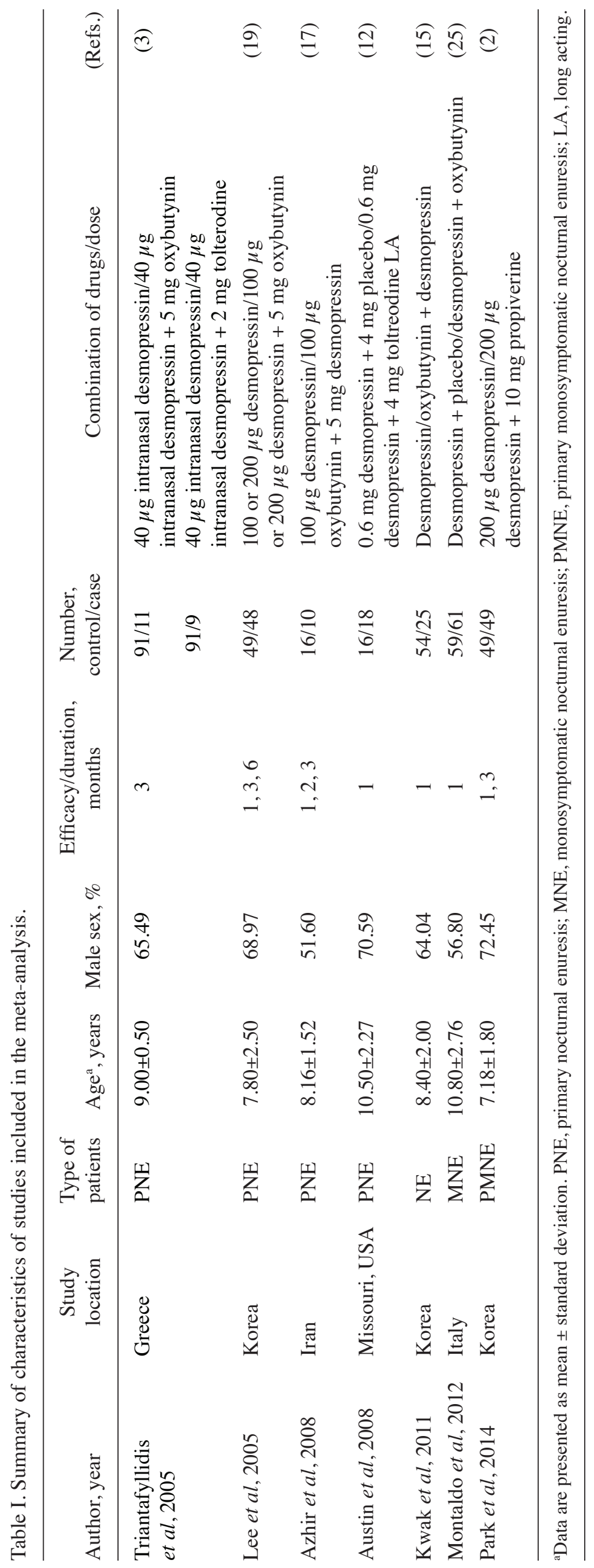



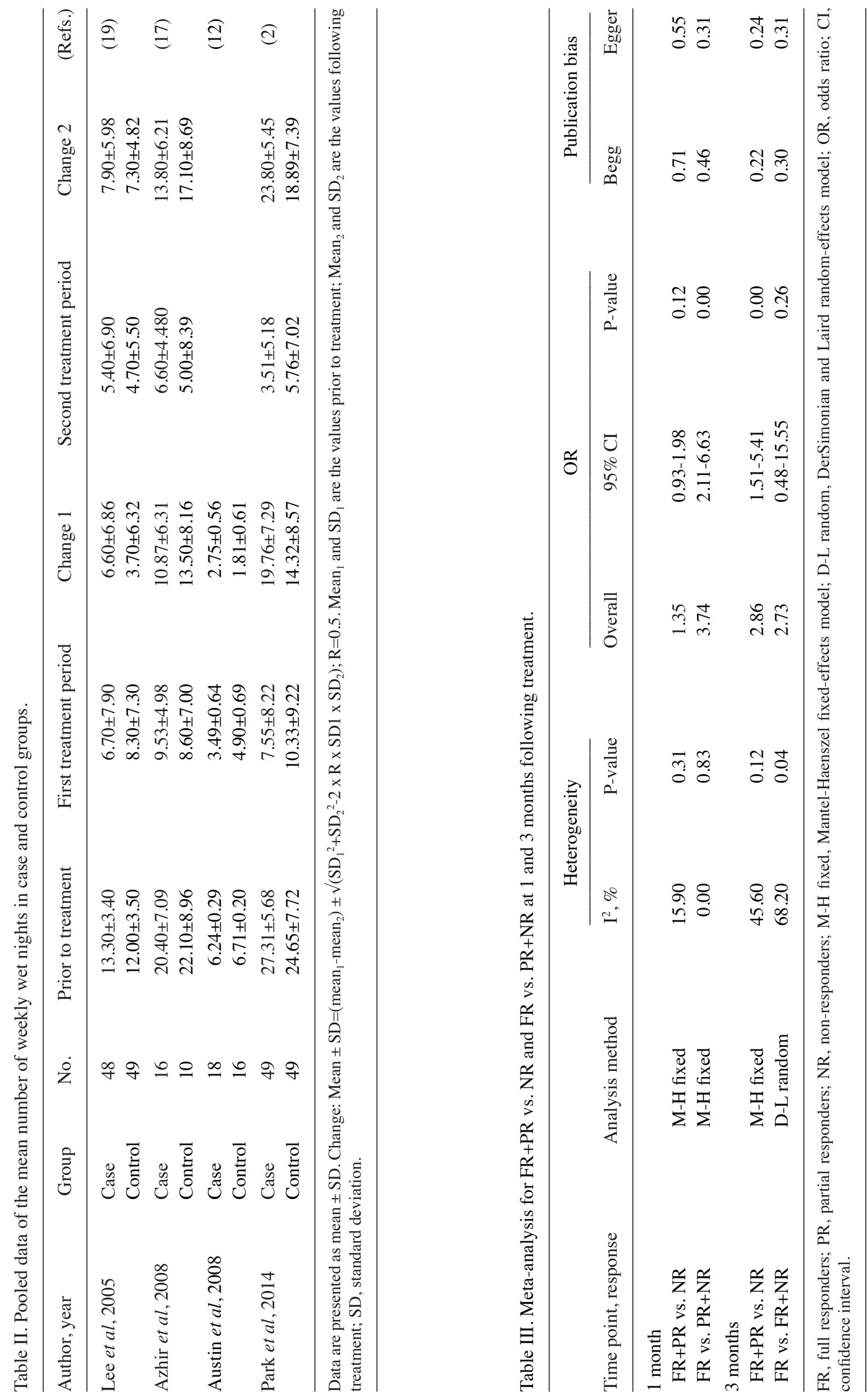


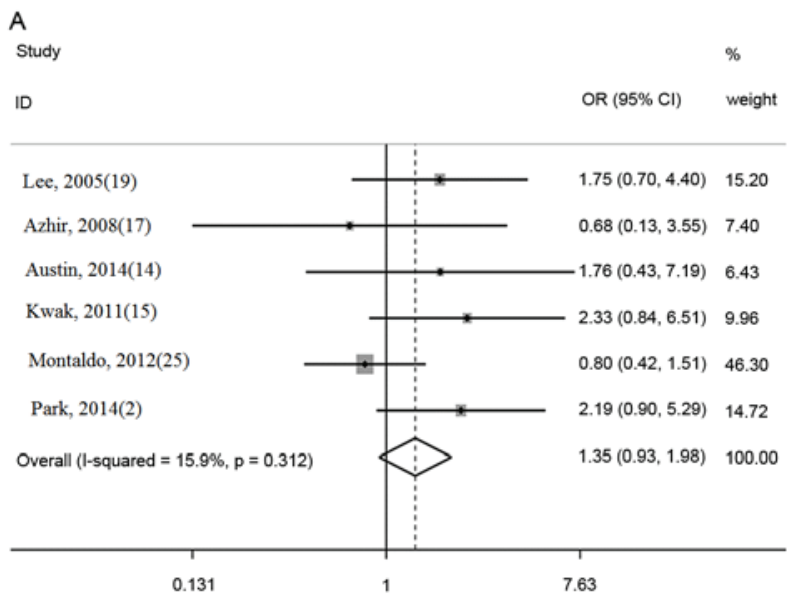

B

Study

ID $\quad$ OR $(95 \% \mathrm{Cl}) \quad$ Weight

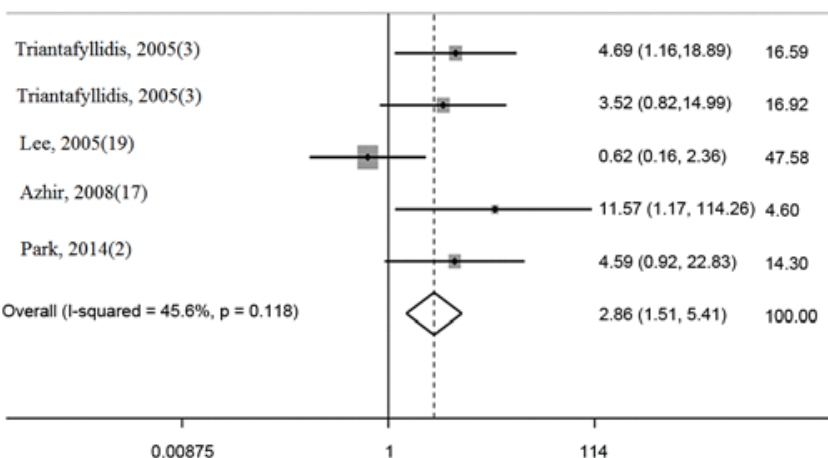

Figure 2. Forest plot of the evaluation of full responders + partial responders vs. non-responders following (A) 1 month and (B) 3 months of treatment. OR, odds ratio; $\mathrm{CI}$, confidence interval.

A

Study

ID
B

$\% \quad$ Study

OR $(95 \% \mathrm{Cl}) \quad$ Weight $\quad$ ID
OR $(95 \% \mathrm{Cl})$ Weight
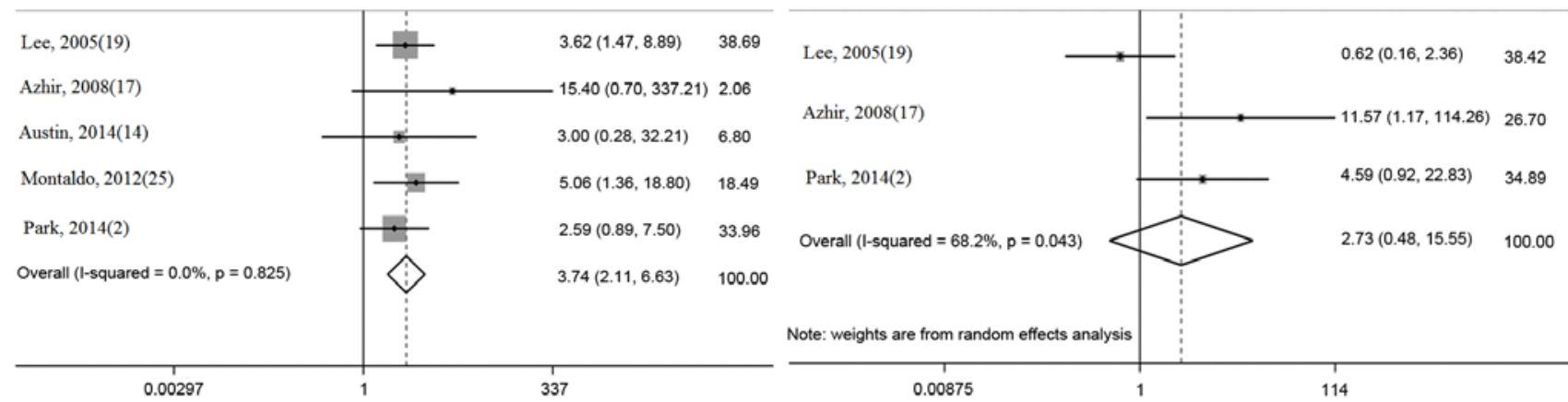

Figure 3. Forest plot of the assessment of full responders vs. partial responders + non-responders following (A) 1 month and (B) 3 months of treatment. OR, odds ratio; $\mathrm{CI}$, confidence interval.

for FR vs. PR+NR, it may be speculated that, compared with the monotherapy, for the short-term treatment (1 month), the combination therapy resulted in more FR and fewer PR; for the long-term treatment ( 3 months), more PR were achieved with the combination therapy while the proportion of FR was similar for the two therapies.

Evaluation of the change in the mean number of wet nights following 1 and 3 months of treatment. For analysis of the change in the mean number of wet nights, 4 eligible studies were included for 1 month and 3 eligible studies were included for 3 months of treatment. The primary results are presented in Table IV. The D-L random-effects model was applied for the calculation of SMD and 95\% CI due to significant heterogeneity being observed ( 1 month, $\mathrm{I}^{2}=59.20 \%$; 3 months, $\mathrm{I}^{2}=59.20 \%$ ). Following 1 month of treatment, the SMD was 0.72 (95\% CI: 0.28-1.15; P=0.00; Table IV and Fig. 4A), suggesting that there was a significant difference in the change in the mean number of wet nights between the combination therapy and monotherapy, and the change in the patients treated with combination therapy was greater than that in the patients treated with monotherapy at 1 month after treatment. Following 3 months of treatment, the SMD was 0.44 (95\% CI: -0.02-0.89; $\mathrm{P}=0.06$; Table IV and Fig. 4B). Although no significant difference was observed in the change of the mean number of wet nights between the combination therapy and monotherapy, the mean number of wet nights was greater following the use of combination therapy. Analysis of the change in the mean number of wet nights indicated that, the change for the combination therapy was more marked compared with that for the monotherapy when the treatment duration was 1 month, and the change was most evident for the short-term combination treatment.

Evaluation of adverse events. For the evaluation of adverse events, 3 eligible studies were included. The M-H fixed-effects model was used to calculate the overall $\mathrm{OR}$ and $95 \% \mathrm{CI}$ as no significant heterogeneity was detected $\left(\mathrm{I}^{2}=0.00 \% ; \mathrm{P}=0.68\right)$. The overall OR was 4.07 (95\% CI: 1.00-16.59, P=0.05; Fig. 5), which indicated that there was no significant difference in adverse events between the two therapies and suggested that adding an anticholinergic agent to the first-line desmopressin regimen for the treatment of NE would not lead to more adverse effects. 
Table IV. Meta-analysis for change in the mean number of wet nights.

\begin{tabular}{|c|c|c|c|c|c|c|c|c|}
\hline \multirow[b]{2}{*}{ Time point } & \multirow[b]{2}{*}{ Analysis method } & \multicolumn{2}{|c|}{ Heterogeneity } & \multicolumn{3}{|c|}{ SMD } & \multicolumn{2}{|c|}{ Publication bias } \\
\hline & & $\mathrm{I}^{2}, \%$ & P-value & SMD & $95 \% \mathrm{CI}$ & P-value & Begg & Egger \\
\hline 1 month & D-L random & 59.20 & 0.06 & 0.72 & $0.28-1.15$ & 0.00 & 0.73 & 0.50 \\
\hline 3 months & D-L random & 59.20 & 0.09 & 0.44 & $-0.02-0.89$ & 0.06 & 1.00 & 0.95 \\
\hline
\end{tabular}

SMD, standardized mean difference; CI, confidence interval; D-L random, DerSimonian and Laird random-effects model.

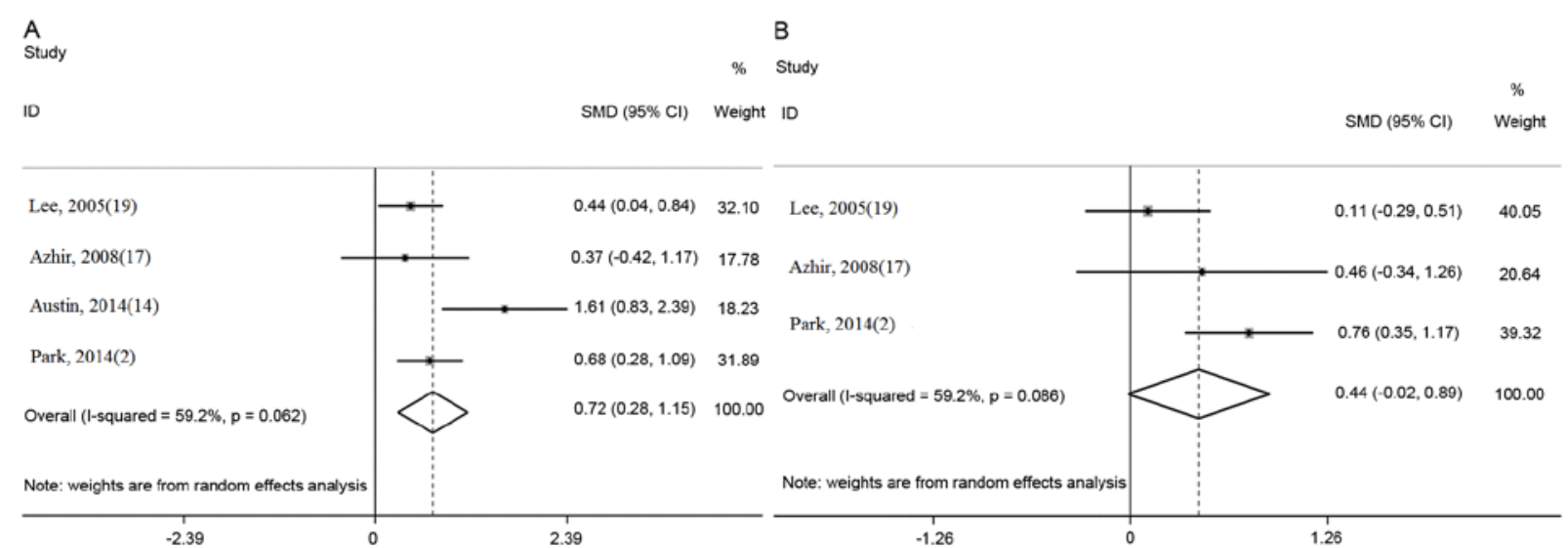

Figure 4. Forest plot of evaluation of the change in the mean number of wet nights following (A) 1 month and (B) 3 months of treatment. SMD, standardized mean difference; $\mathrm{CI}$, confidence interval.

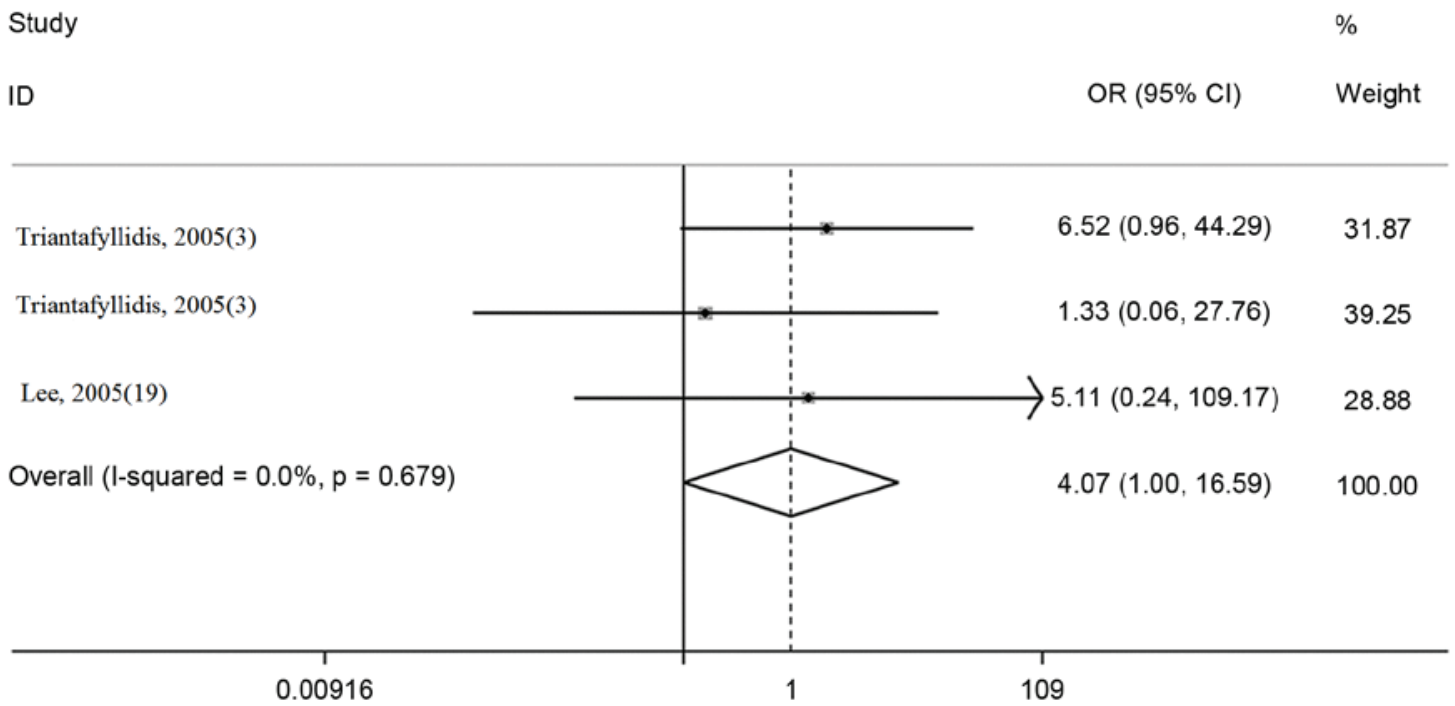

Figure 5. Forest plot of the estimation of adverse events. OR, odds ratio; CI, confidence interval.

Publication bias. In the present meta-analysis, Begg's test and Egger's test were used to examine the publication bias and the results for outcomes and change in the mean number of wet nights are presented in Tables III and IV, respectively. The P-values in these analyses were $>0.05$, indicating the absence of publication bias. A lack of publication bias was also indicated for adverse events (Begg's test, $\mathrm{P}=1.00$; Egger's test, $\mathrm{P}=0.50$ ). The corresponding funnel plots in Figs. 6 and 7 also demonstrate that there was no publication bias in the current meta-analysis.

\section{Discussion}

In order to comprehensively evaluate the clinical efficacy and safety of combination therapy comprising desmopressin plus an anticholinergic agent compared with desmopressin alone for the treatment of NE in children, the present study conducted a meta-analysis including 8 eligible studies. From consideration of the results of FR+PR vs. NR, and FR vs. $\mathrm{PR}+\mathrm{NR}$ analyses it was speculated that, compared with 
A

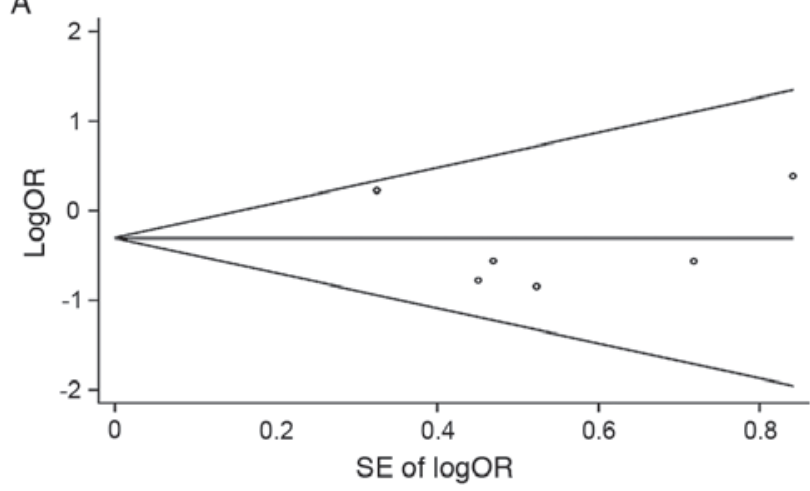

C

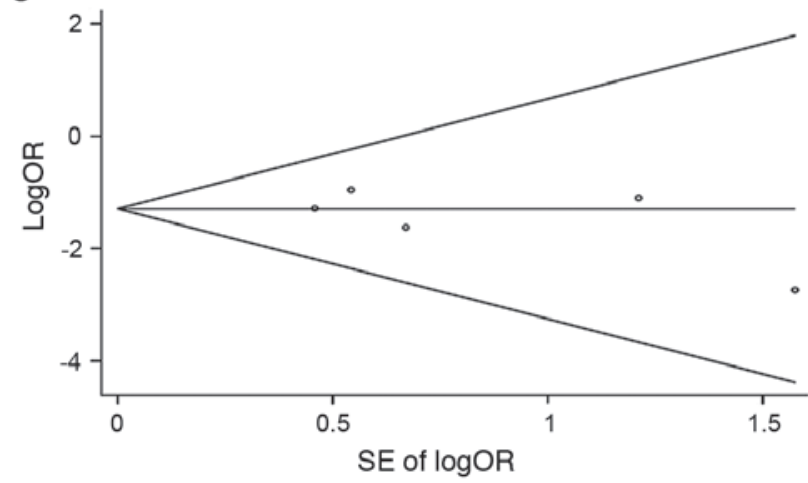

B

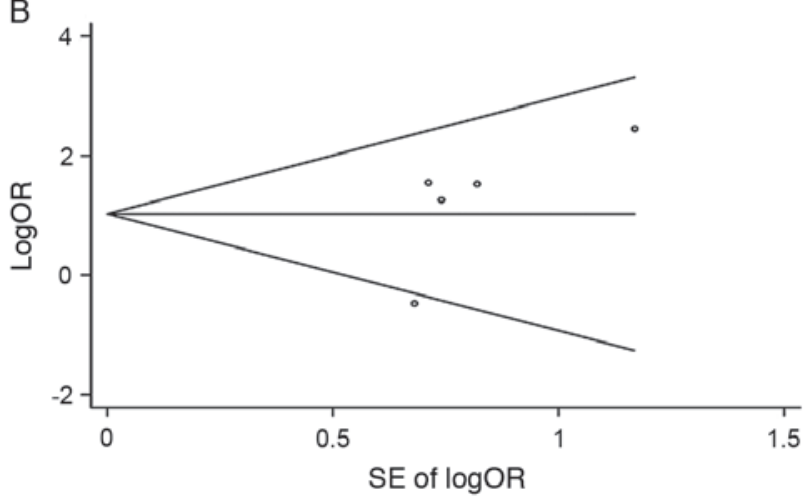

D

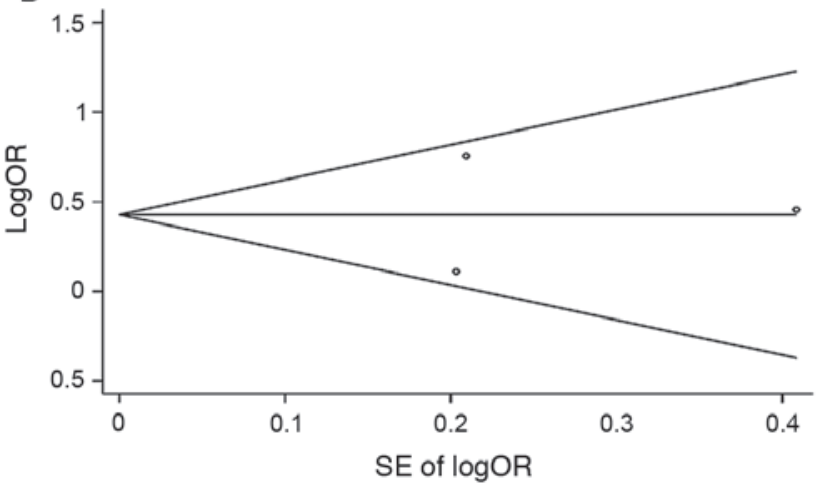

Figure 6. Funnel plots for evaluation of FR+PR vs. NR following (A) 1 month and (B) 3 months of treatment and for evaluation of FR vs. PR+NR following (C) 1 month and (D) 3 months of treatment. FR, full responders; PR, partial responders; NR, non-responders; logOR, natural logarithm of the odds ratio; SE, standard error.
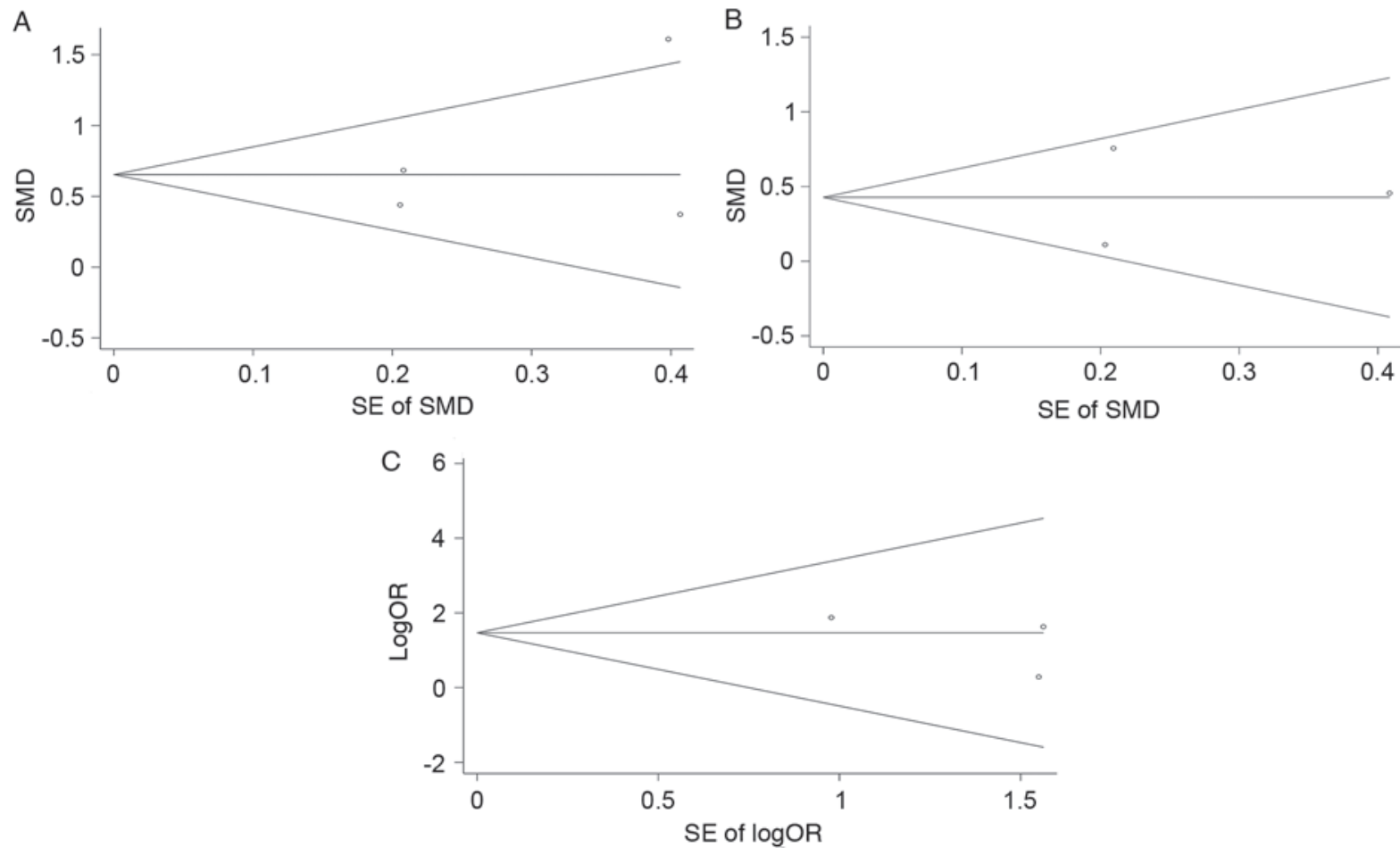

Figure 7. Funnel plots for investigation of the change in mean number of wet nights following (A) 1 month and (B) 3 months of treatment and (C) adverse events. SMD, standardized mean difference; logOR, natural logarithm of the odds ratio; SE, standard error.

the monotherapy, for the short-term treatment (1 month), the combination therapy resulted in more FR and fewer PR; for the long-term treatment (3 months), more PR occurred in the combination therapy group with an equivalent proportion of FR between groups. This may partially explain why FR was notably improved, compared with PR+NR at 1 month while 
FR+PR was improved compared with NR at 3 months in terms of evaluating the effect of the combination therapy. For the change in the mean number of wet nights, the results indicated that compared with the monotherapy, the change was more marked in the combination therapy when the treatment duration was 1 month and the change was more evident with the short-term combination treatment.

The results of the efficacy demonstrated that the combination therapy comprising desmopressin plus an anticholinergic agent was more effective compared with desmopressin monotherapy for the treatment of NE in children. For adverse events, the OR was 4.074 and no significant difference was detected between the combination therapy and monotherapy, which suggested that the combination therapy did not lead to more adverse events when used for the treatment of NE in children. Considering efficacy and the safety, the present study indicates that compared with desmopressin monotherapy alone, the combination therapy comprising desmopressin plus an anticholinergic agent was more effective with equivalent safety in children with NE.

Although NE is not a direct threat to a patients' life, it imposes psychological suffering during daily life for children with NE and their parents (6). A number of theories including genetics and neurological issues have been proposed as reasons for children failing to respond to their full or contractive bladder during sleep $(3,26)$. No single explanation has been accepted widely, as NE is a multiple pathophysiological disorder characterized by a mismatch between urine production at night and the nocturnal bladder capacity (3). The desmopressin monotherapy, which is generally regarded as a relatively benign medication, has been recommended as the first-line treatment for enuresis by the ICCS $(6,27)$. However, the clinical efficacy of desmopressin alone is not always satisfactory. Anticholinergic therapy has been demonstrated to promote the response to desmopressin by increasing the functional bladder capacity; therefore, a combination therapy comprising desmopressin plus an anticholinergic agent has been administered to children who experience no or partial responses to desmopressin monotherapy. Furthermore, Montaldo et al (25) suggested that these combined therapies have an association with a restricted bladder capacity and thickened bladder wall, which may be good predictors for clinical treatment. A multicenter study published in 2014 indicated that, compared with oral desmopressin alone, a combination of desmopressin and an anticholinergic was more effective and quicker in reducing PMNE (2). A clinical trial performed by Lee et al (19) indicated that for children with NE, MNE or PSNE, the combination therapy of desmopressin plus oxybutynin is equally effective and is significantly more rapid and efficacious than desmopressin alone (19). To the best of our knowledge, the current study is the first meta-analysis to comprehensively evaluate the efficacy and safety of the combination therapy of desmopressin plus an anticholinergic agent compared with desmopressin alone for the treatment of $\mathrm{NE}$ in children.

However, a number of limitations should be considered when interpreting the results. Firstly, with regard to desmopressin administration, patients in certain studies were treated with oral desmopressin, while others were administered desmopressin intranasally. As more data becomes available, subgroup analysis stratified by the administrative route of desmopressin may be performed. Secondly, the dosage of desmopressin was not exactly the same in the 8 covered studies, and the dosage effect requires evaluation when more data are available. Additionally, the present analysis was not able to compare the relapses of the two therapies due to insufficient data being available.

In conclusion, the present meta-analysis indicates that, compared with desmopressin monotherapy, combination therapy using desmopressin plus an anticholinergic agent is more effective for the treatment of NE. With regard to safety, the introduction of an anticholinergic agent into the desmopressin monotherapy does not cause more adverse events. Therefore, compared with desmopressin monotherapy, the combination therapy is more effective with equivalent safety for children with NE.

\section{References}

1. Bayne AP and Skoog SJ: Nocturnal enuresis: An approach to assessment and treatment. Pediatr Rev 35: 327-334; quiz 335, 2014.

2. Park SJ, Park JM, Pai KS, Ha TS, Lee SD and Baek M; Korean Children's Continence and Enuresis Society: Desmopressin alone versus desmopressin and an anticholinergic in the first-line treatment of primary monosymptomatic nocturnal enuresis: A multicenter study. Pediatr Nephrol 29: 1195-1200, 2014.

3. Triantafyllidis A, Charalambous S, Papatsoris AG, Papathanasiou A, Kalaitzis C, Rombis V and Touloupidis S: Management of nocturnal enuresis in Greek children. Pediatr Nephrol 20: 1343-1345, 2005.

4. Robson WL: Clinical practice. Evaluation and management of enuresis. N Engl J Med 360: 1429-1436, 2009.

5. Van Herzeele C, De Bruyne P, Evans J, Eggert P, Lottmann H, Norgaard JP and Vande Walle J: Safety profile of desmopressin tablet for enuresis in a prospective study. Adv Ther 31: 1306-1316, 2014.

6. Kaneko K: Treatment for nocturnal enuresis: The current state in Japan. Pediatr Int 54: 8-13, 2012.

7. Van Herzeele C, Evans J, Eggert P, Lottmann H, Norgaard JP and Vande Walle J: Predictive parameters of response to desmopressin in primary nocturnal enuresis. J Pediatr Urol 11: 200. e1-e8, 2015

8. Cederblad M, Sarkadi A, Engvall G and Nevéus T: No effect of basic bladder advice in enuresis: A randomized controlled trial. J Pediatr Urol 11: 153.e1-e5, 2015.

9. Neveus T, Eggert P, Evans J, Macedo A, Rittig S, Tekgül S, Vande Walle J, Yeung CK and Robson L; International Children's Continence Society: Evaluation of and treatment for monosymptomatic enuresis: A standardization document from the International Children's Continence Society. J Urol 183: 441-447, 2010.

10. Faraj G, Cochat P, Cavailles ML and Chevallier C: Treatment of isolated nocturnal enuresis: Alarm or desmopressin? Arch Pediatr 6: 271-274, 1999 (In French).

11. Ahmed AF, Amin MM, Ali MM and Shalaby EA: Efficacy of an enuresis alarm, desmopressin, and combination therapy in the treatment of Saudi children with primary monosymptomatic nocturnal enuresis. Korean J Urol 54: 783-790, 2013.

12. Austin PF, Ferguson G, Yan Y, Campigotto MJ, Royer ME and Coplen DE: Combination therapy with desmopressin and an anticholinergic medication for nonresponders to desmopressin for monosymptomatic nocturnal enuresis: A randomized, double-blind, placebo-controlled trial. Pediatrics 122: 1027-1032, 2008.

13. Kiddoo D: Nocturnal enuresis: Non-pharmacological treatments. BMJ Clin Evid 2015: pii: 0305, 2015.

14. Austin PF, Bauer SB, Bower W, Chase J, Franco I, Hoebeke P, Rittig S, Vande Walle J, von Gontard A, Wright A, et al: The standardization of terminology of lower urinary tract function in children and adolescents: Update report from the Standardization Committee of the International Children's Continence Society. J Urol 191: 1863-1865.e13, 2014.

15. Kwak KW, Park KH and Baek M: The efficacy of enuresis alarm treatment in pharmacotherapy-resistant nocturnal enuresis. Urology 77: 200-204, 2011. 
16. Butler RJ, Robinson JC, Holland P and Doherty-Williams D: Investigating the three systems approach to complex childhood nocturnal enuresis-medical treatment interventions. Scand J Urol Nephrol 38: 117-121, 2004.

17. Azhir A, Gheissari A, Fragzadegan Z and Adebi A: New treatment protocol for primary nocturnal enuresis in children according to ultrasound bladder measurements. Saudi Med J 29: $1475-1479,2008$

18. Telli O, Samanci C, Sarici H, Hascicek AM, Kabar M and Eroglu M: Can urinary nerve growth factor and bladder wall thickness correlation in children have a potential role to predict the outcome of non-monosymptomatic nocturnal enuresis? J Pediatr Urol 11: 265.e1-e5, 2015.

19. Lee T, Suh HJ, Lee HJ and Lee JE: Comparison of effects of treatment of primary nocturnal enuresis with oxybutynin plus desmopressin, desmopressin alone or imipramine alone: A randomized controlled clinical trial. J Urol 174: 1084-1087, 2005.

20. Higgins JP, Thompson SG, Deeks JJ and Altman DG: Measuring inconsistency in meta-analyses. BMJ 327: 557-560, 2003.

21. Leonard T and Duffy JC: A Bayesian fixed effects analysis of the Mantel-Haenszel model applied to meta-analysis. Stat Med 21: 2295-2312, 2002.
22. Brockwell SE and Gordon IR: A comparison of statistical methods for meta-analysis. Stat Med 20: 825-840, 2001.

23. Senn S: Trying to be precise about vagueness. Stat Med 26: 1417-1430, 2007.

24. Light RJ and Pillemer DB: Summing up: The science of reviewing research. Harvard University Press, Cambridge, MA, 1984.

25. Montaldo P, Tafuro L, Rea M, Narciso V, Iossa AC and Del Gado R: Desmopressin and oxybutynin in monosymptomatic nocturnal enuresis: A randomized, double-blind, placebo-controlled trial and an assessment of predictive factors. BJU Int 110: E381-E386, 2012.

26. Sarici H, Telli O, Ozgur BC, Demirbas A, Ozgur S and Karagoz MA: Prevalence of nocturnal enuresis and its influence on quality of life in school-aged children. J Pediatr Urol 12: 159.e1-e6, 2016.

27. DiBianco JM, Morley C and Al-Omar O: Nocturnal enuresis: A topic review and institution experience. Avicenna J Med 4: 77-86, 2014. 